Horizons philosophiques

\title{
La citoyenneté en France entre particularisme et universalisme
}

\section{Sophie Duchesne}

Volume 12, numéro 1, automne 2001

Langue : identité plurielle

URI : https://id.erudit.org/iderudit/801196ar

DOI : https://doi.org/10.7202/801196ar

Aller au sommaire du numéro

Éditeur(s)

Collège Édouard-Montpetit

ISSN

1181-9227 (imprimé)

1920-2954 (numérique)

Découvrir la revue

\section{Citer cet article}

Duchesne, S. (2001). La citoyenneté en France entre particularisme et universalisme. Horizons philosophiques, 12(1), 87-108.

https://doi.org/10.7202/801196ar d'utilisation que vous pouvez consulter en ligne.

https://apropos.erudit.org/fr/usagers/politique-dutilisation/ 


\section{La citoyenneté en France entre particularisme et universalisme1}

Selon Michael Walzer : «A citizen is, most simply, a member of a political community, entitled to whatever prerogatives and encumbered with whatever responsibilities are attached to membership2». Le mot "citoyen" peut aussi être défini à partir de ses deux antonymes : l'étranger d'abord, celui qui n'appartient pas à la communauté en cause; mais aussi le sujet. Le citoyen est un acteur qui, d'une façon ou d'une autre, dispose d'un accès au pouvoir. L'étude de la citoyenneté s'articule autour d'une question essentielle : le consentement à l'obligation est-il possible pour tous? Autrement dit, dans quelles mesures, dans quelles conditions et selon quelles modalités est-il possible de donner à tous ceux sur lesquels s'exerce un pouvoir démocratique, les moyens de consentir ou non aux décisions prises par ce pouvoir?

La citoyenneté semble un concept facile à définir, même si les questions qu'elle pose sont difficilement solubles. Mais de ce fait, les travaux sur la citoyenneté portent sur des domaines de recherche extrêmement divers; et quiconque se lance aujourd'hui ne serait-ce que dans une recherche bibliographique ou un travail d'approche sur la citoyenneté, acquiert rapidement le sentiment qu'ayant attrapé un petit bout de fil, il est en train de ramener à lui toute la pelote, à savoir, toute la science politique! ${ }^{3}$ Au point que certains auteurs commencent à s'interroger sur l'intérêt de travailler plus avant avec ce concept. Ainsi Will Kymlicka et Wayne Norman, dans leur survey article, "Return of the Citizen»4, concluent : «Here, as with the other issues we have examined in this survey, it remains unclear what we can expect from a "theory of citizenship". "lls montrent en effet comment, au cours des années 90 , le champ d'étude couvert par la citoyenneté s'étant exagérément étendu, les oppositions

1. Cet article, qui constitue la version française d'un texte publié en anglais dans la Revue Tocqueville (vol. XX no 1, 1999), présente les principales conclusions de mon travail de doctorat, publié dans l'ouvrage : Citoyenneté à la française, Paris, Presses de Sciences Po, 1996.

2. Michael Walzer, "Citizenship" in Political innovation and conceptual change, Terence Ball, James Farr and Russell L. Hanson eds., Cambridge, Cambridge U.P., 1989, ch. 10, p. 211.

3. Le reader's publié par Bryan Turner et Peter Hamilton, Citizenship. Critical concepts (London and New York, Routledge, 1994) avec ses deux volumes et ses 52 contributions, donne un bon aperçu de l'étendue des questions prises en compte.

4. Will Kymlicka et Wayne Norman : «Return of the Citizen : a Survey of Recent Work on Citizenship Theory", in Ethics, 104(2), janvier 1994, p. 352-381. 
structurant les positions des différents auteurs étant devenues récurrentes et les opinions s'étant largement consolidées les unes par rapport aux autres, ce concept ne leur paraît plus très heuristique pour la science politique ou, plus précisément, pour la théorie politique.

L'idée que le concept de citoyenneté n'est pas - ou n'est plus, ou est insuffisamment - opérationnel est partagée par ceux qui remarquent que, malgré l'ampleur des sujets abordés dans ce cadre, certaines catégories de travaux font défaut, en particulier ceux qui portent sur les représentations - que je qualifierai «d'ordinaires" - de la citoyenneté. On commence à bien connaître ce que les savants, les théoriciens pensent de la citoyenneté, comment il faut la définir et ce qu'elle devrait être en pratique, les institutions, mais aussi les rôles et les qualités qu'elle requiert. Beaucoup de travaux aussi sont consacrés à ce que le débat public, autour des questions de maîtrise des flux migratoires et d'attribution de la nationalité, révèle des conceptions de la citoyenneté partagées dans différents pays 5 . Mais on sait extrêmement peu de choses sur ce que les citoyens «ordinaires", ceux qui ne participent pas directement à ce type de débats, pensent de ce qu'ils sont en tant que tels, de leur(s) rôle(s) de citoyen, des qualités dont ils croient avoir besoin pour l'assumer et du statut qui devrait permettre tout cela.

L'article de Robert Lane, intitulé : "The Tense Citizen and The Casual Patriot : Role Confusion in American Politics" figure d'exception dans ce domaine. C'est en 1991 seulement qu'ont été publiés les premiers résultats d'une autre enquête - comparative, cette fois, en Grande-Bretagne et aux États-Unis - sur les représentations ordinaires de la citoyenneté7. Mais sur la France, rien de tel n'avait encore été publié.

5. Le livre de Roger Brubaker, Citizenship and Nationhood in France and Germany (Harvard University Press, Cambridge Mass. 1992) et en particulier les chapitres consacrés à la période récente, est typique de ce genre d'approche. Voir aussi le livre d'Adrian favell, Philosophy of Integration. Immigration and the Idea of Citizenship in France and Great Britain, London, Macmillan, 1997.

6. Paru in The Journal of Politics, $N^{\circ} 27,1965, p .736$. Cette analyse est fondée sur une enquête réalisée en 1965, enquête qualitative portant sur dix-sept hommes blancs, «middle class», vivant sur la Côte Est des États-Unis. Robert Lane s'est entretenu très longuement avec chacun d'eux (puisque les entretiens ont parfois dépassé une dizaine d'heures), à partir d'un questionnaire semi-directif très détaillé visant à lui permettre de comprendre comment et pourquoi des citoyens ordinaires pensent ce qu'ils pensent en matière politique. Une partie de l'entretien portait plus spécifiquement sur la représentation que chacune des personnes interrogées se faisait des rôles et des qualités d'un citoyen idéal, et sur l'évaluation qu'elle fait de ses propres "performances" en la matière.

7. "The Nature of Citizenship in the United States and Great Britain : Empirical Comments on Theoretical Themes"; P.J.Conover, I.M.Crewe and D.D.Searing, The Journal of 
L'enquête, dont je vais présenter les principaux résultats, est une enquête qualitative réalisée dans le cadre d'une thèse de doctorat en science politique. L'objectif, à l'origine, était de s'inscrire dans la lignée des travaux inspirés par les hypothèses d'Alessandro Pizzorno sur les "collectivités identifiantes" permettant de mieux comprendre et mieux expliquer la constitution du rapport au politique des individus ${ }^{8}$. La citoyenneté semblait une notion propre à faire apparaître la diversité des contours de ces collectivités; mais ce travail n'a pas été sans surprise dès lors que l'une des caractéristiques de la citoyenneté "ordinaire» est d'être finalement largement à l'écart du politique ${ }^{9}$.

\section{Être citoyen, c'est assumer ses liens avec ses concitoyens.}

La citoyenneté "ordinaire», même si elle se décrit à travers plusieurs modèles, peut être définie par une formule unique : "être citoyen c'est assumer ses liens avec ses concitoyens". Mais les qualités et les rôles impliqués par cette définition dépendent directement de la façon dont sont conçus les liens entre concitoyens : suivant qu'on les envisage comme des liens naturels, concrets, inscrits dans une histoire, ou suivant qu'ils apparaissent comme des liens fortuits, artificiels, définis par des frontières administratives, deux modèles de citoyenneté se dessinent, la première qu'on appellera "citoyenneté par héritage», la seconde, "citoyenneté par scrupules".

Cette définition fait une faible part au politique. Ici, la citoyenneté se décrit plutôt dans le champ de ce que la science politique étudie sous l'angle de la civilité. C'est le rapport avec les autres qui définit la citoyenneté "ordinaire», et non pas le rapport avec le pouvoir. Comment interpréter ce décalage? Chacune des personnes rencontrées lors de cette enquête a clairement exprimé le sentiment de n'avoir, seule, aucun accès, direct, personnel, au pouvoir, et donc aucun moyen, pour revenir à la

Politics, vol. 53, no 3, August 1991. Pamela Conover, Ivor Crewe et Donald Searing ont réalisé des entretiens de groupe pour faire apparaître les différentes conceptions de la citoyenneté dans ces deux pays et voir dans quelle mesure elles confirment ce que les théoriciens de la citoyenneté disent d'elles. Car les auteurs considèrent que les raisons du relatif épuisement de la discussion conceptuelle sont aussi à rechercher du côté de la faiblesse - pour ne pas parler du manque - des travaux empiriques dans ce domaine.

8. Cf. "Sur la rationalité du choix démocratique", in Sur l'individualisme, Pierre Birnbaum et Jean Leca dir., Paris, Presses de la FNSP, 1986, p. 330-369.

9. Les entretiens, réalisés auprès d'une quarantaine de personnes choisies pour leur diversité en termes de propriétés sociales (mais pas de résidence, puisqu'elles vivaient toutes à Paris et en région parisienne), étaient des entretiens dits "non-directifs", recueillis à partir de la consigne "est-ce que vous voulez bien qu'on parle de ce que c'est pour vous Bqu'être un citoyen, de tout ce que représente pour vous le fait que vous soyez, vous, un citoyen?" et exploités à l'aide d'une méthode qualitative et typologique, visant à reconstituer les modèles culturels de citoyenneté mêlés dans les entretiens. 
définition dont nous sommes partis, de consentir, ou non, aux décisions qui l'obligent. Par contre, chacune des personnes interrogées sait aussi que sa force, face au pouvoir, repose sur le nombre. Si un citoyen a du pouvoir, en tant que tel, en tant que citoyen (et non en fonction de ses attributs personnels), c'est parce que les citoyens, ensemble, sont (tout) puissants ${ }^{10}$. Le pouvoir de chaque citoyen dépend des autres, de ses concitoyens. C'est donc la relation qu'il entretient avec ses concitoyens qui définit les limites de son pouvoir. C'est ainsi que l'on peut comprendre comment la citoyenneté "ordinaire" se décrit à travers les liens, à travers la façon de valoriser et d'entretenir ces liens avec les autres. Mais des différentes façons dont sont conçus les liens qui unissent les citoyens dépendant différents modèles de citoyenneté.

10. Une partie des entretiens a été recueillie au moment où tombait le mur de Berlin. Les manifestations en Allemagne de l'est sont évoquées par plusieurs des personnes interrogées. 
(24)

NOUS NE SAVONS PLUS TRĖS BIEN CE QUE NOUS DEVONS TRANSMETTRE

FACE À DES ETRANGERS JE ME SENS RESPONSABLE DE L'IMAGE DE LA FRANCE

(6)

ON EST FORCÉMENT

CITOYEN D'UN PAYS: IL FAUT BIEN ÊTRE DE QUELQUE PART

I

(4)

J'AI DES RACINES QUELQUE PART EN FRANCE C'EST CONCRET

$\stackrel{\varphi}{9}$

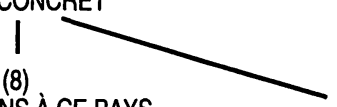

J'APPARTIENS À CE PAYS J'EN SUIS RESPONSABLE PAR TOUT MON COMPORTEMENT

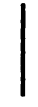

(9)

NOUS AVONS DES DEVOIRS

C'EST CERTAIN. IL N'Y A

PAS QUE DES AVANTAGES

(10)

IL FAUT S'ENGAGERI

C'EST TROP FACILE DE CRITIQUER SANS RIEN FAIRE I

IL FAUT VOTER

MOI J'AI TOUJOURS VOTÉ
(5)

ON NE RENONCE PAS

A SON HÉRITAGE

À SA NATIONALITÉ

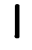

(3)

JE ME SENS UN PEU

L'HÉRITIER DE

L'HISTOIRE DE FRANCE

NOUS SOMMES TOUS

ENGAGÉS ENSEMBLE DANS LA MARCHE DU PAYS
।

(25)

BIENTÔT ON NE POURRA

PLUS ÊTRE FRANCAIS.

IL FAUDRA ÊTRE EUROPÉEN<smiles>[CH]C</smiles>

(1)

ÉTRE CITOYEN IC

EN FRANCE

C'EST ÉTRE FRANÇAIS

I

(2)

NOUS PARTAGEONS

UNE LANGUE, DES COUTUMES,

UNE CULTURE

NOUS AVONS UN PATRIMOINE

EN COMMUN

1

(15)

$\begin{array}{ll}\text { NOUS NOUS } & \text { LA FRANCE, C'EST GRAND } \\ \text { RESSEMBLONS } & \text { MAIS SA COMMUNE ON }\end{array}$

(13)

NOUS SOMMES SOLIDAIRES

COMME DANS LA VIE

DE FAMILLE

(14)

NOUS DEVONS NOUS

GARDER DE

LA DIVISION
MAIS SA COMMUNE, ON

PEUT S'Y INTEGRER

IL Y A DES CHOSES QUI SE

PERDENT: LES FRANÇAIS

SONT DE PLUS EN PLUS

INDIVIDUALISTES

TOUT A CHANGÉ

LA VIE ÉVOLUE UN PEU

TROP VITE POUR NOUS

(21)

IL YADE PLUS EN PLUS

D'ÉTRANGERS. PARFOIS

JE NE ME SENS PLUS

TOUT A FAIT CHEZ MOI

I

(19)

LES ÉTRANGERS ONT

POUR SE SENTIR

IL FAUT S'INTÉGRER

SOUVENT DU MAL

A S'INTÉGRER

(20)

LES ÉTRANGERS SONT

LES BIENVENUS ICI S'ILS

J'AIME LA FRANCE

JE M'Y SENS CHEZ MOI

AIMENT MON PAYS 


\section{La citoyenneté par "héritage»}

Ce premier modèle de citoyenneté, appelé «citoyenneté par héritage" est celui qui s'articule autour d'une conception «naturaliste» si on peut dire, des liens entre concitoyens. II fonctionne sur une assimilation plus ou moins directe entre citoyenneté et nationalité. Ici, un citoyen est d'abord un Français.

Ce modèle s'organise autour de quelques mots : le temps, le sol et la famille, qui structurent le schéma. Le temps forme l'ossature du schéma, la citoyenneté s'organise entre le passé, qui la fonde, le présent dans lequel elle s'exerce, et l'avenir où elle court le risque de se perdre. Le sol est à la fois le support sur lesquels s'inscrivent les repères - dans les entretiens les plus proches du modèle de «l'héritage», on rencontre fréquemment le vocabulaire des racines, des repères, des frontières, des limites, ou encore la notion d'ancrage; et le sol est aussi la substance même de l'héritage, du patrimoine, cette terre de France que les concitoyens possèdent ensemble. Elle est en même temps le théâtre du temps qui passe, le sol et le temps apparaissant comme étroitement liés. Ce premier modèle de citoyenneté se décrit par la métaphore familiale : la famille est le lien dans le temps et dans l'espace, à la fois modèle dont dérivent les formes de la solidarité nationale et cause de cette solidarité. C'est parce qu'on appartient à une famille, c'est parce que les membres de cette famille, ceux des générations antérieures, ont souffert pour faire ce pays comme il est vrai que leurs héritiers, les citoyens d'aujourd'hui, sont ce qu'ils sont et ont les moyens d'exercer dignement leur citoyenneté. Le lien national, qui constitue l'essentiel du lien civique, n'est en somme qu'une extension du lien familial, auquel, idéalement, il emprunte les formes.

Ce modèle se caractérise par une insistance mise sur le concret, le vécu, par opposition à l'abstrait et l'intellectuel, qui apparaissent comme des artifices. Parler de sa citoyenneté, parler de soi en tant que citoyen, c'est parler de soi en vrai, ce n'est pas échanger des idées; c'est dire ce que l'on est «pour de vrai». Être citoyen, être Français renvoie à un sentiment d'appartenance réel, concret, profond. Répétons-le, l'identité nationale telle qu'elle est exprimée dans ces entretiens n'a rien d'une construction intellectuelle ${ }^{11}$.

11. En ce sens, la citoyenneté "ordinaire" contredit les conclusions savantes de Dominique Schnapper, qui écrit : "La notion même de nation ethnique est contradictoire dans ses termes. C'est l'effort d'arrachement aux identités et aux appartenances vécues comme naturelles par l'abstraction de la citoyenneté qui caractérise en propre le projet national. II existe une seule idée de nation." (in La communauté des citoyens. Sur l'idée moderne de nation, Paris, Gallimard, 1994, p.24) II est tout à fait clair que, pour ceux qui assimi- 
La famille est au coeur de ce modèle, et qui dit famille, dit amour. Cet amour est d'abord celui que le citoyen porte à ses ancêtres, sans qui il ne serait pas - c'est une simple question de faits, une question "naturelle". II est empreint de la reconnaissance que le citoyen leur adresse, et qu'il adresse à ce qu'ils ont créé, à sa nation. II tire sa force du respect de leurs souffrances, de l'admiration pour ce qu'ils ont contribué à bâtir. Les entretiens les plus proches de ce modèle livrent souvent, en guise d'explication, de justification des devoirs du citoyen, cette formule : «mes (nos) ancêtres se sont battus pour ça!».

L'amour porté à ceux qui ont fait le citoyen ce qu'il est se prolonge dans la sympathie pour tous ceux qui sont, eux aussi, issus de gens qui se sont battus pour faire ce pays; le sentiment qui unit des compatriotes est donc un élément de "l'héritage", il ne suppose pas qu'ils se reconnaissent des qualités particulières. Les personnes dont l'entretien contribue le plus à ce modèle de citoyenneté jouent cependant volontiers avec les stéréotypes nationaux : «les Français ne sont pas mieux que les autres" disent-ils, "ils sont moins travailleurs et disciplinés que les Allemands, ils n'ont pas, comme les Italiens, le sens du beau, etc." Les Français aussi ont leurs défauts, mais ces défauts ont leurs bons côtés puisqu'ils contribuent à caractériser et à distinguer les Français des autres citoyens. L'amour de ses ancêtres et la sympathie à l'égard de ses concitoyens autorisent le citoyen à s'aimer lui-même. Ce transfert réflexif du respect, voire de l'admiration, fait la force de cette conception de la citoyenneté.

Cette citoyenneté-là est une identité, en ce sens que, se disant citoyen français, celui qui parle dit effectivement qui il est : il est le produit particulier d'une histoire qui s'est déroulée à un endroit particulier, sur un sol défini, délimité par des frontières. Le citoyen est bien sûr individuellement particulier, au sens où il a une histoire personnelle qui le distingue de tout le monde; mais le fait d'être inscrit dans une histoire collective le rend à la fois suffisamment semblable à ceux qui sont inscrits dans la même histoire et suffisamment différent de ceux qui ne le sont pas pour qu'il puisse considérer que cette différence entre lui et les autres, entre nous et les autres nous, est une différence naturelle, même si les frontières elles-mêmes ne le sont pas. Cette particularité ne lui pèse pas, il en est fier, voire il s'en enorgueillit. Elle lui donne une consistance, une force qui le dépasse et le protège. Le national, dans ce modèle-là, tire sa force de l'identification à sa nation. L'histoire dont il est le produit a une pesan-

lent citoyenneté et lien national, l'une et l'autre n'ont rien d'abstrait, et la nation est vécue comme une identité ou une appartenance naturelle. 
teur, une signification intrinsèques qui le soulagent en partie d'avoir à justifier sa propre existence par ses réalisations personnelles. D'un certain point de vue, les siècles l'ont fait pour lui'2. C'est en ce sens qu'une des personnes interviewées s'exclame : «La tempête peut souffler, pour moi, ça se passe bien, tant que mes racines sont bien ancrées dans le sol». En échange d'une relative impuissance sur le cours des choses puisque, en somme, c'est à l'échelle de l'histoire que les choses prennent sens : à ce niveau, le citoyen ne peut rien, il n'est rien - il bénéficie de la force, de la puissance de sa nation, celle du tout dont il est partie.

Ce modèle de citoyenneté est bien connu, ne serait-ce qu'en France même, d'autant qu'il fait la synthèse de deux "sous-modèles", l'un qui prolonge la conception catholique traditionnelle de la France, et l'autre qui provient de la conception républicaine de ce pays. Dans le premier cas, l'identification à la France renvoie à une histoire immémoriale commencée dans la nuit des temps : c'est la France de toujours, la France éternelle, celle qui autrefois s'appelait la Gaule, et depuis s'est poursuivie sans que son identité, sa persistance n'aient été mises en cause. L'autre sousmodèle renvoie à une autre conception de la France : le pays de la Révolution, la nation des petits luttant contre les gros, celle qui a tranché la tête des rois et déclaré les droits de l'homme. $\mathrm{Si}$, dans le premier sousmodèle, la xénophobie est assez présente, les personnes dont l'entretien contribue le plus au second sous-modèle expriment au contraire haut et fort leur souci de ne pas sombrer dans le racisme. La France dont elles se réclament est une nation qui s'est constituée dans la lutte, une nation inscrite dans une dynamique de l'histoire, et dont le projet est ouvert à ceux qui veulent $y$ contribuer. Mais elles insistent sur le temps qu'il faut à une personne pour se fondre dans le destin national : une vie au moins, si ce n'est une génération. La nation évolue comme une famille, grâce au mariage, à l'apport régulier de sang neuf. Mais il faut de la volonté, de part et d'autre, pour que l'entrée dans la famille soit une réussite.

Ces deux sous-modèles, appelés "national» et «républicain», contribuent donc à confondre dans le modèle de "l'héritage" deux courants de pensée que la science politique appréhende le plus souvent sur le mode du conflitit3. Car, aussi divergentes qu'ils puissent apparaître sur le plan

12. Plusieurs personnes manifestent alors une certaine commisération pour ces Américains, citoyens "sans histoire", ou dotée d'une histoire si brève, impropre à justifier, au même titre que l'histoire de France, l'existence de ceux qui en sont issus.

13. Sur le plan de la sociologie historique, Yves Déloye a retracé la longue lutte entre les conceptions catholiques et républicaines de la citoyenneté (cf. Ecole et citoyenneté. L'individualisme républicain de Jules Ferry à Vichy : controverses, Paris, Presses de la Fondation nationale des sciences politiques, 1994). En sociologie politique, Guy Michelat et Michel Simon ont montré la prégnance de l'héritage catholique sur l'orienta- 
des valeurs, ils convergent sur une représentation du citoyen comme membre d'un tout, d'une nation, inscrit dans une histoire, entre passé, présent et avenir, évoluant dans un monde caractérisé par les frontières qui servent de repères à l'humanité. En ce sens, la tradition catholique et la tradition républicaine se retrouvent dans une vision du monde holistique et particulariste.

Ce modèle frappe aussi par sa proximité avec la fameuse définition qu'Ernest Renan a donnée de la nation : "Une nation est une âme, un principe spirituel. Deux choses qui, à vrai dire, n'en font qu'une, constituent cette âme, ce principe spirituel. L'une est dans le passé, l'autre dans le présent. L'une est la possession en commun d'un riche legs de souvenirs; l'autre est le consentement actuel, le désir de vivre ensemble, la volonté de continuer à faire valoirs/'héritage "qu'on a reçu indivis. L'homme, Messieurs, ne s'improvise pas ${ }^{14}$ ". L'idée que la valeur du citoyen dépend du passé dont il est l'héritier et du soin qu'il met à le perpétuer est tout entière contenue dans la dernière phrase de ce texte. Elle est récurrente dans les entretiens les plus proches de ce modèle. On est Homme parce qu'on est héritier, on est homme parce que des gens vous ont précédé, ont peiné en prévision de votre existence; rien ne se cré par génération spontanée. On ne peut que porter, continuer et transmettre ce qui vous a été donné.

Le modèle de "l'héritage" diffère cependant du texte de Renan par la forte incertitude qui pèse sur l'avenir. Le texte de Renan est un texte offensif, qui vise notamment à justifier les prétentions françaises sur l'Alsace et la Lorraine. En comparaison, les entretiens, même les plus proches de ce modèle, apparaissent singulièrement en retrait. Renan pose la légitimité de la nation française dans un "vouloir vivre» en commun qui correspond en fait à un "vouloir poursuivre" en commun. Or le modèle de «l'héritage" - comme le montrent les propositions 21 à 25 du schéma correspondant - intègre l'idée qu'il représente la fin d'une conception. De ces entretiens sur la citoyenneté, les plus proches de "l'héritage" sont souvent les plus denses, les plus beaux (en termes littéraires); ils sont souvent lyriques, mais d'un lyrisme triste, nostalgique. Face à la conviction que "être citoyen aujourd'hui en France, c'est être

tion politique et l'antagonisme qu'elle entretient avec la tradition de gauche à laquelle ressortit notamment la tradition républicaine (cf. Classe, religion et comportement politique, op.cit.).

14. E.Renan, Qu'est-ce qu'une nation? Conférence faite en Sorbonne le 11 mars 1882 , citée ici d'après l'édition présentée par Joël Roman, Paris, Presses Pocket, coll. Agora, 1992 (p.54). II va de soi que la correspondance entre le modèle et le texte de Renan n'est pas le résultat d'un processus d'influence direct. Sur les trente-huit personnes interrogées, moins d'une dizaine sont susceptibles d'avoir lu ce texte. Pour ma part, je n'en ai vraiment pris connaissance, dans le détail, qu'après avoir formalisé le modèle. 
Français", et malgré la certitude que ça ne saurait être autrement ( «il faut bien être de quelque part!»), on sent peser sur ces entretiens une triple menace.

Il y a d'abord la présence, ressentie comme trop pesante, des étrangers en France. Dans sa version républicaine, on l'a vu, le modèle conçoit bien, pour la France elle-même, les bienfaits, voire la nécessité de l'immigration. Mais celle-ci doit être contrôlée, les flux ne doivent être ni trop importants, ni trop rapides, et les étrangers doivent être répartis sur le territoire, de façon à ce qu'ils ne perturbent pas l'équilibre des régions où ils s'implantent et qu'ils puissent en adopter le mode de vie.

II y a ensuite la "menace» européenne, que Renan lui-même avait désignée comme une fin possible des nations ${ }^{15}$. Mais l'Europe n'est pas qu'une menace; elle est aussi le seul rempart vraisemblable contre une disparition totale, brutale, des frontières, qui elle signifierait la fin de tout, de toutes les cultures, de toutes les nations, de toute citoyenneté possible. Mieux valent les frontières de l'Europe que pas de frontières du tout. Après tout, la France s'est formée par l'intégration des régions; l'Europe devrait pouvoir intégrer ses nations, à condition de laisser le temps faire son office. Les Français se sont battus contre les Anglais et les Allemands, l'histoire de l'Europe est faite de ces combats. Mais mieux vaut s'être battus les uns contre les autres que ne s'être jamais rencontrés. Pour ceux dont la citoyenneté s'apparente le plus à ce modèle, ceux donc dont la citoyenneté est à proprement parler une identité nationale, l'Europe est la moindre des menaces qui pèsent sur la citoyenneté française.

Ce sont les Français eux-mêmes qui la mettent le plus en danger, en ne croyant plus à leur nation, en ne faisant plus d'efforts, en n'étant plus prêts à se battre, à se "défoncer" pour elle : "Les Français sont de plus en plus individualistes". Renan avait aussi prédit que les nations ne dureraient que tant qu'elles seraient légitimes. "Tant que cette conscience morale (la nation) prouve sa force par les sacrifices qu'exige l'abdication de l'individu au profit d'une communauté, elle est légitime, elle a le droit d'exister16». Pour les gens les plus proches du modèle de «'héritage», le vrai danger est là, dans la perte de légitimité de la nation, dans l'individualisme - qui pour eux signifie simplement égoïsme - des citoyens de ce pays. Le danger vient donc de ceux qui témoignent d'une autre conception de la citoyenneté, individualiste et universaliste, que résume le modèle dit «des scrupules».

15. "Les nations ne sont pas quelque chose d'éternel. Elles ont commencé, elles finiront. La confédération européenne, probablement, les remplacera.» in Qu'est-ce qu'une nation ?, op.cit. p.55.

16. Qu'est-ce qu'une nation?, op.cit. p.56. 


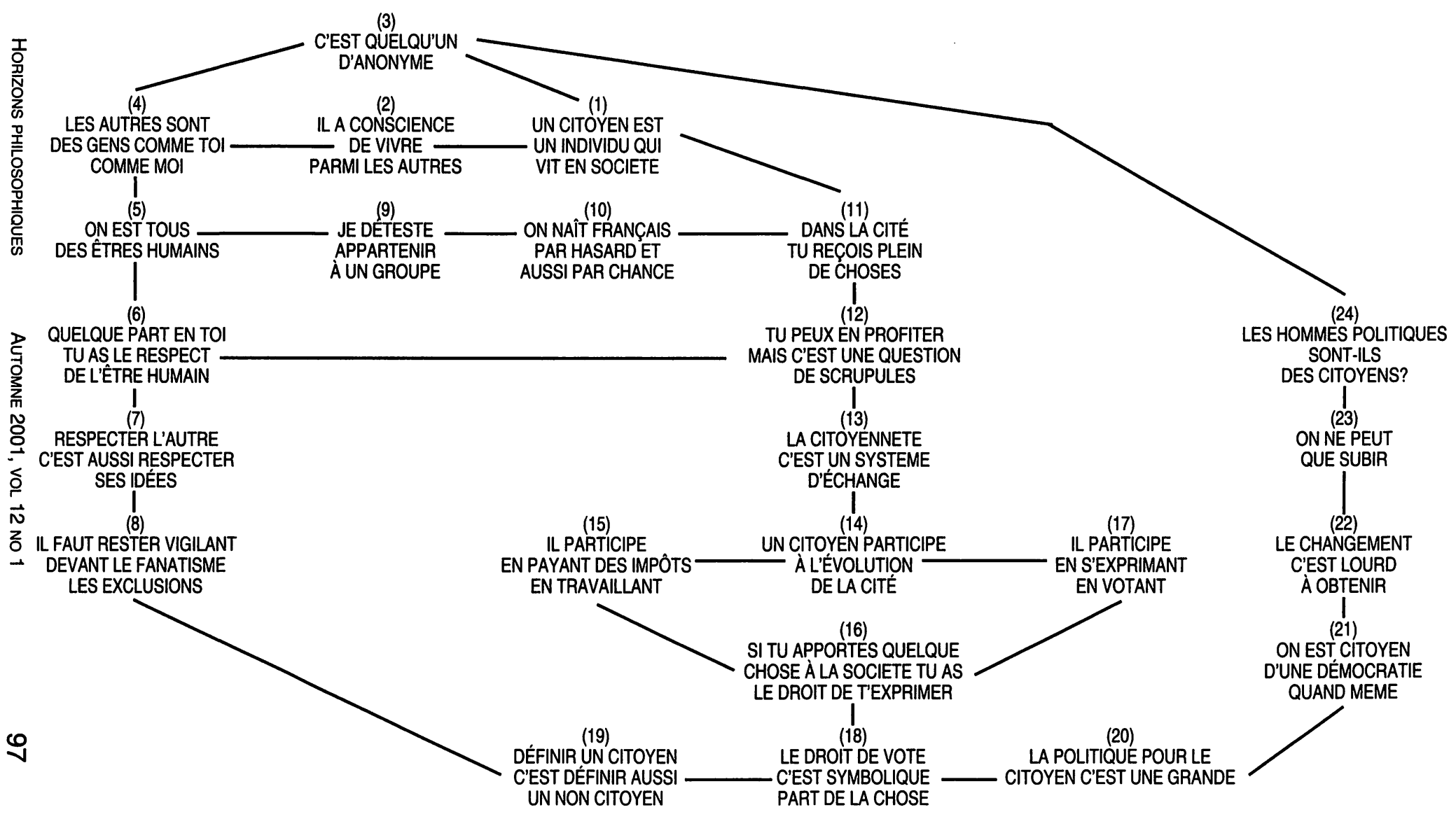




\section{Citoyen par "scrupules»}

Bien que la logique qui l'organise lui soit propre, ce deuxième modèle fonctionne aussi souvent par opposition au modèle précédent. Celui-ci était holistique et particulariste, articulé autour des questions de totalité, d'identification, d'ensemble et de ressemblance; celui-là sera individualiste, au sens où la valeur de l'individu prime sur tout, et universaliste.

Le deuxième schéma peut se lire en deux parties : la première (propositions 1 à 10) correspond au niveau légitime - pour ce modèle - de l'existence des citoyens, celui des individus et des relations interindividuelles. Dans ce second modèle, le citoyen ne (re)connaît que d'autres individus, il les rencontre et échange avec eux en toute indépendance, en toute liberté. Toute la difficulté va être de passer de cet univers à celui des relations organisées à l'échelle de la société et d'y trouver des rôles qui préservent son autonomie et son aptitude à échanger avec n'importe quel individu. La citoyenneté représentera, dans ce second modèle, les efforts que l'individu va devoir faire pour accepter la dimension collective de l'existence humaine à laquelle il se sent, de prime abord, étranger, mais dans laquelle il va essayer de trouver une place. La seconde partie (propositions 11 et suivantes) rend compte de la participation du citoyen au collectif.

Dans l'univers des "scrupules", la prégnance des rapports entre individus se manifeste par la domination du couple de pronoms «je / tu», qui s'oppose au couple «je / nous» prépondérant dans le premier modèle. L'individu existe dans les rapports qu'il établit avec les autres, avec d'autres personnes, chacune appréhendée de façon singulière. Toute rencontre avec une autre personne est un processus de reconnaissance réciproque qui permet à chacune non seulement de reconnaître l'autre, mais aussi, par réflexion, de se reconnaître elle-même. La rencontre introduit à un processus d'identification au singulier, à l'unique, qui suppose une capacité à communiquer, à échanger avec n'importe qui, confirmant chacun dans sa réalité d'être humain, dans ses prétentions à l'universalité17. La notion d'échange contient celle de changement : échanger signifie que l'on accepte l'idée de changer, que ce que l'on reçoit comme expériences, comme émotions - en échange de celles qu'on a données - contribue à

17. La sociologie - et avec elle la sociologie politique - aborde les questions d'identité à travers le processus d'identifications à des collectifs, des groupes, tels que la classe sociale, l'ensemble des personnes de même confession, la nation, la région, le groupe dit "ethnique", etc., laissant à la psychologie l'étude de l'identification à des personnes singulières. Pourtant, la dynamique de ce second modèle, quoique portant sur d'un processus d'identification potentielle permanente à tout être humain rencontré, est bien d'essence sociologique et devrait être étudiée en tant que telle. 
transformer l'individu, à le faire sensiblement différent après l'échange de ce qu'il était avant.

Ce modèle de la rencontre entre des individus uniques qui se reproduit à l'infini suppose une flexibilité très grande. L'image la plus forte de la société, dans ce modèle, est celle de la page blanche, de quelque chose en fait d'indescriptible, de mouvant, sans limites, sans frontière ${ }^{18}$. L'individu n'est lié définitivement à rien par nature; il change, il se transforme en fonction de ses rencontres du moment. II n'est lié que par ses affections. II fuit devant toute idée de groupe et d'appartenance. «Moi je déteste appartenir", disent les personnes les plus proches de ce modèle.

Les frontières qui déterminent un groupe - qu'il s'agisse des frontières officielles posant les limites administratives d'un territoire ou d'un pays, ou qu'il s'agisse des frontières symboliques d'appartenance entre groupes - marquant les limites entre ceux qui se ressemblent, les membres du groupe, et ceux qui sont différents, les étrangers, toutes les frontières apparaissent dans ce modèle-là comme une négation de l'individu. Les gens les plus proches de cette vision du monde parlent de "déformation" pour désigner ces traces que laisse l'éducation, et qui font que chacun reconnaît plus facilement comme ses semblables les membres de sa nation, de sa confession, de sa classe, de sa "race»...; il ne reconnaît pas - ou ne reconnaît plus, si l'on se réfère à l'universalité qui caractérise la page blanche, cette société flexible évoluant au rythme des rapports inter-individuels - les autres, les étrangers, comme équivalents de lui. Le membre d'un groupe n'est plus capable d'échange puisqu'il n'accepte plus l'idée de changer, il n'accepte pas les transformations qui résulteraient de la rencontre avec des gens qui sont extérieurs à son groupe. II croit que les caractéristiques que partagent les membres de son groupe constituent sa différence naturelle avec les autres, qu'elles sont une identité qui ne peut être remise en cause. Alors que la seule identité valide dans le modèle des "scrupules", la seule appartenance concevable est l'humanité. Quiconque se définit autrement, par les groupes auxquels il appartient, a perdu le respect de l'être humain, le respect de l'universel qui est la seule valeur, le seul noyau d'humanité par lequel un individu sait qu'il est homme.

18. À la suite de chaque entretien a été réalisé un test projectif très bref, dans lequel on demandait notamment à la personne interrogée de choisir parmi quatre schémas celui qui lui semblait représenter le mieux la société telle qu'elle la voudrait. Les personnes les plus proches de ce second modèle refusent souvent de choisir et expriment la difficulté qu'elles éprouvent à imaginer une représentation valide de la société. Certains laissent entendre que la représentation la plus fiable est finalement celle de la page blanche. 
Dans le premier modèle, le citoyen respecte ses ancêtres et cela le conduit à respecter le fruit de leurs efforts, à savoir lui-même et ses concitoyens. Ici, on assiste également à un transfert de respect et d'affection, mais il n'a plus à voir avec le groupe national. Seul le respect de chaque être humain permet à un individu, un citoyen, de se respecter lui-même. Rompre la chaîne de la reconnaissance potentielle avec n'importe quel individu, quelles que soient ses origines, signifie rompre la chaîne de reconnaissance qui constitue l'humanité. Car dans l'univers des "scrupules", le groupe produit la masse. Dans les entretiens les plus proches de ce modèle, deux images du collectif dominent : la foule sans but, errant dans les supermarchés, dans le métro, les gens qui se gênent, se marchent sur les pieds les uns des autres sans même se regarder; ou la foule enrôlée, déchaînée, mue seulement par la volonté de tuer, de détruire, l'armée en marche dans laquelle chaque soldat ne sait plus qu'obéir ou le peuple révolté. La transfiguration de ces groupes d'êtres sans volonté en humanité passe par l'effort de chacun pour regarder les autres, les individualiser, les reconnaître.

Dans cette vision du monde, il n'y a donc ni peuple, ni territoire puisqu'il n'y a pas de frontières légitimes pour repérer, délimiter l'espace ni histoire : l'existence humaine est indéterminée. La mémoire de l'individu se borne à ce qu'il a vécu, le reste n'est que "déformation», enrôlement. Pourtant, les personnes rencontrées au cours de cette enquête et qui s'inscrivent de façon dominante dans ce modèle-là savent que le monde dans lequel elles vivent fonctionne différemment. Dès lors qu'elle reconnaissent que le progrès existe, elle conçoivent bien qu'il doit se produire, d'une façon ou d'une autre, une accumulation qui n'est pas que négative, une mémoire qui ne fait pas que " déformer» les êtres humains. II y a donc bien une organisation collective qui procède de et par cette accumulation non seulement pour le bien du plus grand nombre, mais même pour le bien de ceux pour qui le collectif apparaît comme repoussant.

II reste qu'il n'y a pas de place sur la terre pour ceux qui veulent vivre seuls. Si chacun bénéficie du progrès, personne non plus ne peut lui échapper. On n'est redevable que de ce qu'on a choisi ou au moins accepté. Aussi l'individu, moralement, ne doit-il rien à la cité, à la collectivité, quel que soit le bien-être qu'elle lui dispense (l'infrastructure, les routes, voire l'aide sociale...) Par contre, il ne peut pas ne pas reconnaître les efforts de ceux qui travaillent, qui peinent pour produire ce dont il bénéficie. II ne peut pas ne pas reconnaître qu'eux aussi sont des gens comme lui, qu'eux aussi peut-être sentent peser sur eux le poids de la 
société et se révoltent à l'idée de s'y sentir prisonniers. C'est pourquoi il ne peut pas "sans scrupules" - le nom du modèle vient de là, le mot est utilisé dans les entretiens - recevoir le fruit de leurs efforts et ne rien donner en échange.

À la figure du traître - traître à sa famille, à ses ancêtres, à sa patrie, à ce qui fait sa grandeur - qui caractérise l'incivisme dans le premier modèle, le modèle des "scrupules» substitue celle du profiteur, celui qui prend sans rien donner. Et la citoyenneté fonctionne alors comme une tentative d'extrapolation de l'échange qui caractérise les relations entre individus, l'extrapolation de ce type de relations au niveau collectif. De même que l'échange individuel transforme ses acteurs, l'échange au niveau collectif vise essentiellement à faire bouger la société et ses membres, à les transformer, les rendre de plus en plus flexibles, de plus en plus ouverts au changement. La participation s'accomplit, dans ses formes les plus visibles, à travers le travail, le paiement de l'impôt, l'expression des opinions, le vote, etc. Mais - et c'est très important dans ce modèle - puisque chacun est seul à même d'évaluer la valeur qu'il accorde à ce dont il bénéficie et le coût que représente l'effort de prendre part à la collectivité, il détermine seul, et à chaque instant, le niveau et les formes de sa participation. Personne ne peut ni ne doit juger de ce qu'un autre doit donner. Ce modèle manifeste une incapacité totale à penser ce qui est imposé autrement que dans les termes de la contrainte.

Ce modèle connaît lui aussi deux variantes. La citoyenneté des "scrupules" peut se lire comme un pari : que l'être humain prenne pied dans la société, contribue à la rendre meilleure, sans s'y noyer, sans se fondre dans la masse ni perdre son humanité. Peut-on vraiment participer, avoir un engagement à l'échelle du collectif, que ce soit dans le politique ou ailleurs, sans perdre son individualité, sans à un moment ou à un autre se laisser "déformer"? L'image qui vient souvent dans les entretiens les plus proches de ce modèle est celle des militants politiques qui parlent comme le chef de leur parti et ne savent plus que reproduire les idées élaborées au sommet. Le pari de la citoyenneté débouche sur une question : «les hommes politiques sont-ils des citoyens?» La réponse, positive ou négative, à cette question, correspond aux deux sousmodèles : ceux qui pensent que les hommes politiques ont au moins le mérite d'essayer, et que mieux vaut prendre le risque de devenir un apparatchik que de rester un profiteur, seront désignés comme démocrates; ceux qui ne croient pas qu'on puisse échapper à l'attraction des groupes et qui pensent que l'essentiel est de sauver son être sont appelés les spectateurs du monde. 


\section{La «tension» de la citoyenneté}

Ces deux modèles de citoyenneté structurent l'ensemble des entretiens recueillis au cours de cette enquête. Ils semblent souvent antagonistes, mais une présentation plus détaillée permettrait de faire apparaître, à travers les rôles de citoyen notamment, les actes que requiert la morale de chacun des modèles (voter, s'informer, travailler, payer ses impôts...) et qui sont autant de points de passage de l'un à l'autre. D'ailleurs, les visions du monde inscrites dans chacun d'eux ne se développent pas de façon aveugle, dans l'ignorance l'une de l'autre. Les personnes plus proches de l'une des deux conceptions de la citoyenneté savent que leur point de vue ne rend pas complètement compte de la réalité. Les gens qui se situent plutôt du côté de "l'héritage» pensent que leur conception de la nation rend compte d'une réalité dont les autres ne veulent plus, et qu'à terme leur propre conception est condamnée puisque la nation n'existe que si tous, ou presque, y croient. Ceux dont l'entretien est proche des "scrupules" savent aussi que leur rejet des frontières n'est pas unanime et qu'ils devront lutter encore longtemps pour abattre les frontières. Chacun soutient son point de vue, sans ignorer celui contre lequel il peut être conduit à le défendre. Les deux modèles s'opposent au moins autant en logique qu'en valeur. II sont logiquement inconciliables. On ne peut pas penser le citoyen à la fois comme le membre d'un tout ou comme un tout lui-même; il ne peut pas s'inscrire à la fois dans la transmission et dans l'invention, tirer sa force dans le sentiment d'être le fruit des générations et chercher sa grandeur dans sa capacité à inventer, à créer ex nihilo. Ce caractère fondamentalement inconciliable des deux modèles rejoint d'ailleurs l'état de la discussion en science politique : l'opposition entre libéraux et communautaristes fait long feu car elle achoppe sur l'incapacité à concilier logiquement deux principes, l'universalisme et le particularisme (ou l'individualisme et le holisme) ${ }^{19}$.

Mais les modèles présentés ici - peut-être ce point n'a-t-il pas été suffisamment souligné - sont des modèles empiriques et non pas théoriques : ils rendent compte de systèmes de représentations, et non de constructions philosophiques. Ils entretiennent donc des rapports de nature différente. Alors qu'un théoricien affronte nécessairement une exigence de cohérence globale des modèles qu'il élabore, les représentations indi-

19. Sur l'impossible conciliation dans la théorie sociologique du l'holisme et de l'individualisme cf. Norbert Elias, La Société des individus, Paris, Fayard, 1991; sur sa nécessaire conciliation, en matière de citoyenneté en particulier, cf. Jean Leca, "La citoyenneté entre la nation et la société civile", in D.Colas, C.Emeri, J.Zylberberg, Citoyenneté et nationalité. Perspectives en France et au Québec, Paris, PUF, 1991. 
viduelles ne connaissent rien de tel. Rien n'interdit à un citoyen "ordinaire" de penser une chose et son contraire, de se sentir à la fois tout et partie. Alors que les théoriciens doivent choisir entre les conceptions libérale et communautaire, les personnes interrogées peuvent fort bien mêler "héritage" et "scrupules". La typologie "héritage / scrupules» ne correspond d'ailleurs pas à un classement des entretiens - les uns dans le premier modèle, les autres dans le second - . Elle vise à reconstituer les logiques qui structurent les valeurs et les opinions exprimées dans l'ensemble du corpus, chacune de ces logiques rendant compte pour partie de chacun des entretiens. La coïncidence du caractère inconciliable des deux logiques à l'œuvre et de la mixité de fait des représentations recueillies donne naissance à une tension que j'ai appelée, provisoirement, la "tension" de la citoyenneté à la française ${ }^{20}$ puisque la spécificité de la France en la matière reste à vérifier.

20. Cette dénomination renvoie également à l'article pionnier de Robert Lane, "The tense citizen...» op.cit. 
Schéma n०3: la répartition des entretiens en fonction de leur contribution à chacun des modèles

REPUBLICAINS

$\vec{Q}$

DENISE

CITOYEN +

DEMOCRATES

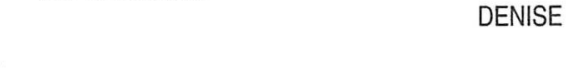

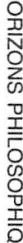

CHANTAL

HERITAGE

FRANCOISE

LOUISE

EDITH

SIDALI

ANNE

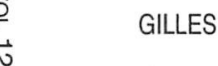

LAURE

ARMAND

MARGUERITE ALICE

ANGELE

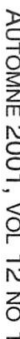

Z LAURE

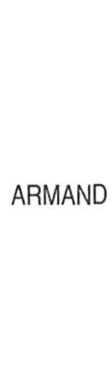

ROSE

JULIETTE

LAURENT

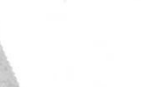

SYLVIE

ERIC

ISABELIE

AGNES

MALIK

ANDRE

FREDERIC

PASCALE 
Le schéma ci-contre représente la contribution de chacun des entretiens aux deux modèles - et aux quatre sous-modèles ${ }^{21}$. II se lit de la façon suivante : plus une personne est située à la gauche du schéma et plus son entretien se rapproche du type idéal que représente le modèle de "l'héritage", et inversement, plus une personne est située à droite de la page et plus son entretien contient de valeurs et de significations propres au modèle des «scrupules". Quant aux personnes situées vers le centre du graphique, leur entretien se caractérise par une forte ambivalence des contributions largement partagées entre les modèles. Celles qui sont représentées dans la zone grisée, au milieu, sont celles chez qui se manifeste au plus haut degré la "tension de la citoyenneté à la française", c'est-à-dire celles dont l'entretien manifeste la difficulté qu'elles éprouvent à affronter la contradiction interne à leur représentation d'ellesmêmes, à vouloir être quelqu'un, une personne unique, universelle, mais quelque part, donc enracinée, particulière. On peut, pour chacune de ces personnes, trouver dans leur trajectoire sociale de quoi fonder des hypothèses sur les causes de la manifestation particulièrement forte de tension. Surtout, on peut a contrario étudier comment les autres parviennent à assumer cette ambivalence fondamentale entre le fait de se sentir à la fois membre d'une entité et totalité à la valeur irréductible.

Les "solutions" auxquelles elles recourent - si tant est que l'on puisse parler de solutions, dès lors que le problème en cause, l'antagonisme logique irréductible entre les deux façons de penser sa citoyenneté, n'apparaît pas nécessairement comme tel aux personnes interrogées - ne sont donc pas des solutions valides au plan théorique, mais seulement des "moyens", des formules permettant de passer, en cours d'entretien, d'un univers de représentations à l'autre en contournant la difficulté logique. Elles consistent en trois «figures» de citoyenneté, qui vont être seulement esquissées.

La première, "être citoyen c'est avoir des droits et des devoirs", reprend une formule que chacune des personnes interrogées a eu l'occasion d'apprendre au cours de ses années d'école, et qui crée un lien entre l'univers des droits et celui des devoirs. Or on peut montrer que les devoirs ne prennent tout leur sens que dans l'univers de "l'héritage" : ils en sont la contrepartie directe, la compensation apportée aux efforts des générations antérieures. Ils sont nécessaires pour préserver ce qui a déjà

21. Je ne commenterai pas ici les effets de la distribution entre les sous-modèles (qui apparaît dans la répartition verticale du schéma), et qui correspond aussi à une distribution entre valorisation et dévalorisation du mot citoyen. Pour plus de détails sur l'élaboration et l'interprétation de ce schéma, qu'il me soit permis de renvoyer au livre Citoyenneté à la française, op.cit., p.180 et ss. 
été accompli. Dans cette vision du monde, il n'y a pas vraiment de droits, seulement des avantages que la nation peut consentir aux siens tant que sa situation le permet; dès qu'un danger pèse sur la nation, tous les avantages peuvent être mis en cause, tout peut être exigé des citoyens, jusqu'au sacrifice de leur vie. Autrement dit, l'idée de droits fondamentaux, intangibles, attachés à l'espèce humaine, qui fonde le modèle des «scrupules", n'a pas de sens dans celui de "l'héritage». À l'inverse, la notion de devoir n'a pas de consistance dans l'univers des "scrupules", puisque l'individu n'a pas la liberté de vivre seul et de refuser toute participation au collectif. Seuls ses droits, et plus encore l'égalité de ses droits avec ceux de tous les êtres humains, sont inviolables. L'utilisation de cette formule, qui fleure bon l'éducation civique, "un citoyen a des droits et des devoirs", permet à l'entretien de basculer d'un modèle à l'autre, d'autant plus facilement que la formule paraît incontestable, puisque familière.

La deuxième figure, "la citoyenneté, c'est le vote» parvient au même résultat en condensant en un même acte, celui qu'accomplit le citoyen en glissant son bulletin dans l'urne, des significations empruntées à chacun des modèles. D'un côté le vote met en scène l'appartenance à la communauté nationale : on vote d'abord parce qu'on en a le devoir, parce que les générations précédentes se sont battues pour qu'on puisse le faire. Le vote est l'acte minimum qui exprime l'engagement de chacun dans sa communauté, le désir de faire quelque chose ensemble, on vote tous ensemble, le même jour, de la même façon. Le vote est alors un acte très peu "politique", au sens partisan : le citoyen a le devoir de voter, mais en gardant son choix secret, puisque qui dit choix dit opposition, conflit. Du côté des "scrupules", au contraire, l'important n'est pas d'aller voter, mais d'avoir le droit. Le droit de vote manifeste la reconnaissance a priori des efforts de chaque citoyen pour participer; il donne corps, par l'égale valeur reconnue à chaque vote, à l'idée d'égalité en valeurs des opinions. Refuser le droit de vote à quelqu'un équivaut à l'exclure, à lui signifier l'absence d'importance ou de reconnaissance qu'on accorde à ce qu'il apporte et pourrait apporter à la collectivité. Mais dans cet univers, l'important est le droit de vote, et non le vote lui-même, dans la mesure où on sait bien que de toute façon une voix ne change rien au résultat du scrutin lequel d'ailleurs ne change peut-être pas grand'chose à l'état de la société elle-même... Aussi, pour qui veut vraiment participer, y a-t-il des choses plus importantes à faire que d'aller voter. La réduction symbolique de la citoyenneté au vote permet elle aussi de basculer d'un univers de significations à l'autre, de passer du citoyen comme membre d'une communauté nationale au citoyen comme membre d'une communauté universelle. 
La dernière de ces figures de citoyenneté est un peu particulière, dans la mesure où elle est sans doute surdéterminée par la période à laquelle les entretiens ont été recueillis, à savoir, la célébration du bicentenaire de la Révolution 22 "Être citoyen en France c'est être héritier de la déclaration des droits de l'homme et du citoyen" permet aussi de combiner, sur un mode d'ailleurs plus intégré que les deux figures précédentes, l'idée particulariste d'héritage avec celle, universaliste, de Déclaration des droits. Être citoyen en France, c'est être héritier de cette communauté politique à la française et de son l'histoire; or ce qui fait la grandeur de cette histoire, c'est le fait que des Français se sont battus pour des principes universels et en particulier, pour la Déclaration des droits de l'homme.

\section{Conclusion}

Avant de conclure, je voudrais d'abord souligner un intérêt du travail monographique : il permet de restituer la diversité des systèmes de représentations au sein d'une entité culturelle, et notamment nationale, alors que la comparaison postule presque nécessairement l'homogénéité des systèmes culturels étudiés. Non seulement tous les Français n'ont pas la même conception de leur citoyenneté, mais leurs représentations sont, ainsi qu'elles l'ont été au XIXe siècle, l'objet d'une forte tension entre des visions du monde antagonistes.

La prise en compte de la complexité des représentations françaises ne dispense pas de s'interroger sur leur spécificité. À défaut d'un corpus directement comparable, l'analyse des rares travaux portant sur les représentations de la citoyenneté aux États-Unis et en Grande Bretagne ${ }^{23}$ montre que c'est moins l'existence d'un antagonisme entre conceptions particulariste et universaliste qui caractérise la citoyenneté «à la française" que la force de cet antagonisme. Le caractère exclusif, en France, de la dimension nationale dans le système d'appartenance et la difficulté à penser le pluralisme des appartenances qui en résulte opposent de façon frontale, sans autre solution possible, appartenance à la nation ou absence d'appartenance et avive donc la tension dans les représentations de la citoyenneté.

22. Nombreuses étaient les personnes interrogées à disposer d'un exemplaire de la Déclaration des droits et des devoirs et à s'y référer, mais il apparaissait clairement que l'acquisition en était récente et tenait au contexte de célébration du bicentenaire.

23. Pour une comparaison détaillée avec les résultats présentés par Robert Lane d'une part ( "The tense citizen..." op.cit.), et surtout par Pamela Conover, Donald Searing et Ivor Crewe, ("The nature of citizenship..." op.cit.) cf. S.Duchesne, "Une esquisse d'analyse comparée de la citoyenneté ordinaire", Revue Internationale de Politique Comparée 1(3), déc. 1994. 
Enfin, l'originalité de l'analyse présentée ici porte moins sur le premier modèle de citoyenneté, «la citoyenneté par héritage», que sur le second modèle, celui de la "citoyenneté par scrupules", qui conduit à une appréhension de l'individualisme radicalement distincte de la logique utilitariste sur laquelle on ramène le plus souvent l'individualisme contemporain. De fait, les personnes rencontrées au cours de cette enquête et qui sont les plus proches de cette vision du monde ne sont ni plus rationnelles et ni plus égoïstes que les autres. Leur conception de la citoyenneté répond dans les deux cas à un impératif moral : assumer ses liens avec ses concitoyens signifie aller jusqu'au bout de ce qui fait la grandeur de l'être humain, que ce soit par l'intégration (dans le premier modèle) ou par une quête acharnée de l'indépendance (selon le second modèle), ce qui implique dans chaque cas de mettre en œuvre volonté et conscience. Quant à la rationalité, ce qui fait agir les citoyens des "scrupules", ce qui les pousse à participer, ce n'est pas l'idée de tirer un avantage direct de leur action, puisque de toute façon ils bénéficient déjà de la collectivité, et ils le savent. L'avantage qu'ils vont tirer de leur participation est d'une nature très particulière : tenter de rendre plus supportable leur vision du monde, "d'apprivoiser" le collectif en parvenant à se concevoir comme individu dans la société. Cette approche de l'individualisme comme processus identitaire 24 nécessaire à mon sens pour comprendre ce qui est vraiment en jeu dans les rapports entre membres de la communauté politique aujourd'hui en France, rejoint la récente multiplication des travaux sur l'altruisme.

\author{
Sophie Duchesne \\ CNRS \\ Maison française d'Oxford
}

24. Dans la lignée des travaux de Norbert Elias. Cf. La société des individus, Paris, Fayard, 1991. 